\title{
Clinical study of infective endocarditis in children: a 3 years experience from MGM Hospital, Warrangal
}

\author{
Pratap $\mathbf{G}^{1}$, Polasa $\mathbf{R}^{2}$ \\ ${ }^{1}$ Dr. Gudi Pratap, Associate Professor, ${ }^{2}$ Dr. Polasa Ramesh, Associate Professor, Department of Pediatrics, Kakatiya \\ Medical College, Warangal, Telangana, India.
}

Address for Correspondence: Dr. Gudi Pratap, Associate Professor, Department of Pediatrics, Kakatiya Medical College, Warangal, Telangana, Email ID :gudipratap@rediffmail.com

\begin{abstract}
Background: The impact of endocarditis in childhood accompanies many cardio-vascular disorders. Infective endocarditis (IE) is the most frequently occurring form of endocardium inflammation. Prevailing among the agents are Str. Viridans, Str. Haemoliticus, Staph. Aureus, Staph Epidermidis, Enterococcus, etc. Objective: This study will explore the outcome of children diagnosed with infective endocarditis for a better guidance in management. Methods: This was a prospective study from warrangal from January 2013 to December 2015. 50 patients with definite IE based on modified Duke's criteria were recruited into the study. Clinical presentation, risk factors, echocardiography and outcome were obtained. Results: A total of 50 IE patients were included within the study. The mean age was $6 \pm 5.45$. Most patients $(80.39 \%)$ were diagnosed within the first week of admission. Staphylococcus aureas was the most common pathogen (38\%) and the mitral valve was predominantly affected (68\%). Complication were common and in hospital mortality remains high (27.3\%). Conclusions: Mortality remains relatively high in children with infective endocarditis. The most common complication is cardiac deficiency (70\%), and pleuropericardial effusions and lung embolism
\end{abstract}

Keywords: Infective endocarditis, Children, Clinical evolution, Complications

\section{Introduction}

Infective endocarditis (IE) continues to be a major challenge in modern medicine. Although the overall incidence of infective endocarditis in the paediatric population is considered to be low, over the last 20 years a rising trend in infective endocarditis has been observed among children. This could be due to several reasons including the availability of improved diagnostic techniques, use of continuous central venous catheters and cardiac implants increasing the risk of infection, and the survival of a greater number of infants with congenital heart disease as a result of improved medical management. Despite major advances in both diagnostic and therapeutic procedures, mortality rates have not changed in the past 25 years [1-6]. It presents acutely with high rates of Staphylococcus aureus infection and complications such as cardiogenic shock and embolization [2-4]. One study from a low middle income region highlighted a high incidence of IE in patients with rheumatic heart disease.

Manuscript received: $10^{\text {th }}$ October 2016

Reviewed: $19^{\text {th }}$ October 2016

Author Corrected: $25^{\text {th }}$ October 2016

Accepted for Publication: $30^{\text {th }}$ October 2016
Infective Endocarditis remains a serious issue in early age. A few factors come to support this worrying statement: the disorder occurrence over the past few years has become more frequent, in relation to the larger number of children with heart operations; protracted and expensive treatment; complications as a result from the disorder, sometimes including a re-operation, and a still high rate lethality.

So the main objective of this study was to prospective analyze the IE evolution and outcome in children in our tertiary care hospital.

\section{Methods}

Study Population- All patients admitted to MGM Hospital, Warangal between January 2013 to December 2015 with definite IE according to modified Duke's criteria were included [5]. 50 IE cases were at the age from 1 to 13 years were selected. This study received ethical approval from the institutional ethics committee. 
Inclusion and exclusion criteria- All patients who were diagnosed with definitive IE, as outlined by the modified Duke criteria were included. According to Duke criteria a definitive clinical diagnosis is made based on the presence of 2 major criteria, 1 major criterion and 3 minor criteria, or by 5 minor criteria. A possible clinical diagnosis is made based on the presence of 1 major criterion and 1 minor criteria or by 3 minor criteria. In cases of recurrent IE, only the index case was included in the present analysis. A history of congenital heart disease, previous cardiac surgery, or the presence of prosthetic graft material was not considered a criterion for exclusion.

The definition for IE complications such as severe valve dysfunction, heart failure, septic shock and embolization are based on contemporary guideline. The type of echocardiography (transthoracic, transoeso-phageal or both), valve involved and presence of vegetation were recorded. The valve involved was determined by the presence of vegetation, abscess and fistula on echocardiogram.

Demographic data consist of age, sex, underlying heart disease, past medical history including previous palliative or corrective surgery for cardiac disease, CHD and surgery for complications of IE, predisposing conditions for bacteremia, complications, outcome and treatments were recorded. Clinical data consist of information regarding the sign and symptoms of patients at the time of admission were noted.

Laboratory data consist of complete blood count (leukocytosis), C-reactive protein (CRP), erythrocyte sedimentation rate (ESR) and microscopic hematuria.

Microbiological data consist of all blood cultures used to evaluate IE obtained by the Microbiology Laboratory of the MGM Hospital and processed by using Automated Bactec 9240.

Echocardiographic data consist of transthoracic echocardiography (TTE) performed to determine the location of vegetation, type of valve infected and cardiac complications. Outcomes measure were in-hospital mortality and discharge alive. Good outcome is defined as patient discharged alive and poor outcome is defined as in-hospital mortality.

Statistical analysis- Obtained data were analyzed using SPSS version 15.0 (SPSS Inc., Chicago, Illinois, USA). For descriptive analysis, measurement of mean and standard deviation were calculated for the numerical data.

\section{Results}

Fifty-four medical records fulfilled the search criteria. Four patients did not fulfill the Duke's criteria for definite IE and were excluded.

In the study group $(n=50)$, the mean age was $6 \pm 5.45$ Standard Deviation (SD). The majority were male $37(74 \%)$ and diagnosed with infective endocarditis within the first week of admission to hospital (table 1).

Table-1: Patients' characteristic and clinical examination findings.

\begin{tabular}{|c|c|}
\hline & $\mathbf{N}(\mathbf{\%})$ \\
\hline Gender & $37(74)$ \\
Boys & $13(26)$ \\
Girls & $5(12)$ \\
Valvular heart disease & \\
Previous mitral valve repair & 1 \\
Secondary to previous IE & 2 \\
Rheumatic valve & 1 \\
Mitral valve prolapsed & 1 \\
\hline Congenital heart disease & $3(6)$ \\
Atrial septal defect & 1 \\
Ventricular septal defect & 1 \\
Truncus arteriosus & 1 \\
\hline
\end{tabular}


Table-2: Complications developing in the course of disorder.

\begin{tabular}{|c|c|}
\hline Complications & Number(N) \\
\hline Heart failure & 7 \\
Pericardial and pleural effusion & 4 \\
Thromboembolism & 3 \\
Mediastinitis & 1 \\
\hline
\end{tabular}

Forty-nine out of 50 patients $(98 \%)$ presented with fever. New murmurs and raised inflammatory markers were clues leading to a diagnosis of IE. None of our patients had immunological manifestation of IE such as Osler's node and Janeway lesions.

All patients had at least one set of blood culture taken during hospitalisation. Staphylococcus is the predominant microorganism followed by streptococcus. Echocardiography was performed in all patients and Vegetations were detected in 24 of the IE cases $(48.27 \%)$, located around the cardiac lesion.

The main complications observed with us are as follows: heart failure in 7 children; pericardial and pleural effusions - in 4 cases; lung or system embolism - in 3 and purulent mediastinitis - 1 case. Complications developed due to infective endocarditis are listed in table 2. Most common complication was cardiac failure. Operative measures were performed in 3 children.

\section{Discussion}

We describe the first series of cases with endocarditis in children from the warrangal region. Overall mortality is high at $27.3 \%$.

Our findings revealed that most patients within this cohort presented without classical signs of infective endocarditis. This is consistent with the findings from The International Collaboration on Endocarditis prospective study in 2009 which showed similar changes in the characteristics of IE [6-9].

This study differs from published literature from the period 1960 to 1980 which documented immunological manifestation in almost $50 \%$ of patients with endocarditis [3-10].

The most common causes of IE were non-cyanotic heart disease. From cyanotic hearth disease, TOF was the most frequent cause as per studies done by Lertsapcharoen et al. in Thailand and some other studies [11,12]. As reported by many studies by improving the survival of CHD and also treatment of rheumatic heart disease in children the causative factors of IE is changed and the feature of the disease in most but not all of developing countries is also changed to that reported in developed countries.[13,14]

Staphylococcus aureus was commonest and right sided valves were mainly affected which was consistent with previous published studies [6-10]. However, several studies have studies the role of different organisms in IE, accordingly Gram-positive organisms, particularly alpha-hemolytic streptococci (Streptococcus viridans), S. aureus and coagulase-negative staphylococci, are the most common offenders. S. aureus is the most common cause of acute bacterial endocarditis [14, $15]$.

Recent microbiological studies showed that the rate of IE due to infections by Streptococcus viridians is decreased and the rate of IE due to staphylococcal infections is increased [16]

Culture negative IE made up to $32 \%$ in this study. This is unusually high in comparison to contemporary data which showed rate of culture negative IE between 14$19 \%$ [1,2]. This trend was last seen in 1980s. [1]. The use of antibiotics prior to hospital admission might contribute to this occurrence.

The leading complication in 7 of the cases was cardiac deficiency. This is explanatory in view of the preceding congenital cardiopathy and the general prejudicial condition of the children. The remaining complications the presence of pericardial and pleural effusions were managed conservatively.

The most dramatic and involving hard effort was our battle to contain the cases of embolism and mediastinitis development in one of the cases, where an operation "on the hot" became necessary. Other authors also inform of dramatic development and fatal outcome of the same and 
other complications - stroke in seven children $[7,8]$. Under the circumstances, despite the appropriate symptomatic and surgical treatment, death rate in children with IE remains high. In our survey we have registered lethality of $27.3 \%$, which corresponds to data from other authors [8].

The most common complication, resulting directly into a lethal outcome is the development of a heavy cardiac deficiency in $70 \%$ of the cases. The most dramatic and hard was the evolution of the disorder in the presence of system and lung embolism, quoted by other authors [911].

The indications for surgical intervention in our study include embolic phenomenon, severe acute mitral regurgitation, large vegetation of more than $1 \mathrm{~cm}$, fungal IE and endocarditis in the presence of congenital heart defect. The survival rate seen in post-surgical patients is consistent with prior studies [14,15], emphasising the need for early intervention when indicated especially when medical intervention has failed.

In this study, the diagnosis of IE was performed using Duke criteria. Though many studies have confirmed the utility of this criteria in this regard, but others reported the limitation of these criteria in diagnosing IE in patients with negative blood culture $[17,18]$.

Some studies evaluated the utility of other minor factors that improve the sensitivity of Duke criteria Piere et al. have reported that inclusion of some factors as minor criteria such as splenomegaly, petechiae or purpuric rashes, microscopic hematuria and a high CRP value $>$ $100 \mathrm{mg} / \mathrm{L}$ would improve the sensitivity of the criteria specially in patients with fever [19].

\section{Conclusion}

IE remains still as a problem for contemporary medicine and in particular pediatric cardiology and cardio surgery. IÅ is a serious disease with lethality in our cases of $29 \%$. The most common complication is cardiac deficiency (70\%), and the hardest one - system and lung embolism. Our study highlights the need for further research into disease patterns amongst different healthcare and population environment to gain maximum yield from resource allocation.

Acknowledgments- We wish to thank all the staff members of Department of Pediatrics, Kakatiya Medical College, Warangal for their kind co-operation during the study.
Funding: Nil, Conflict of interest: None initiated, Perission from IRB: Yes

Ethical Clearance: Obtained from Institutional ethics committee

\section{References}

1. Cabell CH, Jollis JG, Peterson GE, Corey GR, Anderson DJ, Sexton DJ, et al. Changing patient characteristics and the effect on mortality in endocarditis. Arch Intern Med. 2002 Jan14;162(1):90-4.

2. Habib G, Hoen B, Tornos P, Thuny F, Prendergast B, Vilacosta I, Moreillon P, de Jesus Antunes M, Thilen U, Lekakis J, Lengyel M, Müller L, Naber CK, Nihoyannopoulos P, Moritz A, Zamorano JL; ESC Committee for Practice Guidelines. Guidelines on the prevention, diagnosis, and treatment of infective endocarditis (new version 2009): the Task Force on the Prevention, Diagnosis, and Treatment of Infective Endocarditis of the European Society of Cardiology (ESC). Endorsed by the European Society of Clinical Microbiology and Infectious Diseases (ESCMID) and the International Society of Chemotherapy (ISC) for Infection and Cancer. Eur Heart J. 2009 Oct;30(19): 2369-413. doi: 10.1093/eurheartj/ehp285. Epub 2009 Aug 27.

3. Zamorano J, Sanz J, Almeria C, Rodrigo JL, Samedi M, Herrera D, et al. Differences between endocarditis with true negative blood cultures and those with previous antibiotic treatment. The Journal of heart valve disease. 2003;12(2):256-60.

4. Jault F, Gandjbakhch I, Rama A, Nectoux M, Bors V, Vaissier E, Nataf P, Pavie A, Cabrol C. Active native valve endocarditis: determinants of operative death and late mortality. Ann Thorac Surg. 1997 Jun; 63(6): 1737-41.

5. Li JS, Sexton DJ, Mick N, Nettles R, Fowler VG, Jr, Ryan T, et al. Proposed modifications to the Duke criteria for the diagnosis of infective endocarditis. Clin Infect Dis. 2000 Apr;30(4):633-8. Epub 2000 Apr 3.

6. Castillo JC, Anguita MP, Ramirez A, Siles JR, Torres $\mathrm{F}$, Mesa D, et al. Long term outcome of infective endocarditis in patients who were not drug addicts: a 10 year study. Heart. 2000;83(5):525-30.

7. Remadi JP, Habib G, Nadji G, Brahim A, Thuny F, Casalta JP, Peltier M, Tribouilloy C. Predictors of death and impact of surgery in Staphylococcus aureus infective 
endocarditis. Ann Thorac Surg. 2007 Apr; 83(4):1295302.

8. Bozkurt AK, Oztunc F, Akman C., et al. Multiple pulmonary artery aneurysms due to infective endocarditis. Ann Thorac Surg. 2003 Feb;75(2):593-6.

9. Hufnagel G., Pankuweit S., Richter A., et al. The European Study of Epidemiology and Treatment of Cardiac Inflammatory Diseases (ESETCID). First Epidemiological Results Die European Study of Epidemiology and Treatment of Cardiac Inflammatory Diseases (ESETCID) - Erste epidemiologischem Ergebnisse. Herz. 2000; 25: 279-285.

10. Venkatesan C., Wainwright M. Pediatric Endocarditis and Stroke: A Single Center Retrospective Review of SevenCases. Pediatr Neurol. 2008 Apr;38 (4): 243-7.doi:10.1016/j.pediatrneurol.2007. 12.009.

11. Lertsapcharoen $\mathrm{P}$, Khongphatthanayothin $\mathrm{A}$, Chotivittayatarakorn P, Thisyakorn C, Pathmanand C, Sueblinvong V. Infective endocarditis in pediatric patients: An eighteen-year experience from King Chulalongkorn Memorial Hospital. J Med Assoc Thai. 2005;88(Suppl 4):S12-6.

12. Liew WK, Tan TH, Wong KY..Infective endocarditis in childhood: a seven-year experience.

Singapore Med J. 2004 Nov;45(11):525-9.
13. Bitar FF, Jawdi RA, Dbaibo GS, Yunis KA, Gharzeddine W, Obeid M. Paediatric infective endocarditis: 19-year experience at a tertiary care hospital in a developing country. Acta Paediatr. 2000 Apr; 89(4):427-30.'

14. Coward $\mathrm{K}$, Tucker $\mathrm{N}$, Darville $\mathrm{T}$. Infective endocarditis in Arkansan children from 1990 through 2002. Pediatr Infect Dis J. 2003 Dec;22(12):1048-52.

15. Heper G, Yorukoglu Y. Clinical, bacteriologic and echocardiographic evaluation of infective endocarditis in Ankara, Turkey. Angiology. 2002;53:191-7.

16. Khan NU, Farman MT, Sial JA, Achakzai AS, Saghir $\mathrm{T}$, Ishaq $\mathrm{M}$. Changing trends of infective endocarditis. J Pak Med Assoc. 2010 Jan;60(1):24-7.

17. Tissières $P$, Gervaix A, Beghetti M, Jaeggi ET. Value and limitations of the von Reyn, Duke, and modified Duke criteria for the diagnosis of infective endocarditis in children. Pediatrics. 2003 Dec;112(6 Pt 1) : e467.

18. Lamas CC, Eykyn SJ. Blood culture negative endocarditis: Analysis of 63 cases presenting over 25 years. Heart. 2003 Mar;89(3):258-62.

19. Pereira CA, Rocio SC, Ceolin MF, Lima AP, Borlot $\mathrm{F}$, Pereira RS, et al. Clinical and laboratory findings in a series of cases of infective endocarditis. J Pediatr (Rio J) 2003;79:423-8.

\section{How to cite this article?}

Pratap G, Polasa R. Clinical study of infective endocarditis in children: a 3 years experience from MGM Hospital, Warrangal. J PediatrRes.2017;4(01):17-21.doi:10.17511/ijpr.2017.i01.04 